\title{
The Philosophical Connotations of Popular Aerobics: Illustrated by the Case of Lesmills
}

\author{
Yanling Wang \\ Maoguo Wu
}

SHU-UTS SILC Business School, Shanghai University, China

doi: 10.19044/esj.2016.v12n11p65 URL:http://dx.doi.org/10.19044/esj.2016.v12n11p65

\begin{abstract}
This paper applies the introspection method to explore the philosophical connotations of popular aerobics by analyzing the case of Lesmills. It argues that people could maintain self-integrity and experience the sense of existence during popular aerobics exercises. Popular aerobics exercises help people increase their strength and courage substantially in addition to sharpening and deepening their human sensibility. In conclusion, this paper advocates and urges the popularization of aerobics exercises for their profound philosophical values.
\end{abstract}

Keywords: Popular Aerobics, Lesmills, Sports Philosophy

\section{Introduction}

A great deal of research on exercise physiology and exercise psychology indicates that physical activity can significantly promote and improve people's physical health and mental well-being. Therefore, exercise and dance therapy are constantly used as aids for patients in clinical medicine. Dance therapy is a kind of physical activity accompanied by music and combined with the rhythm of body activities. It alleviates muscle tension and eases emotional stagnation of exercisers, and helps them to acquire better body image. Generally speaking, exercise therapy is similar to dance therapy except that exercise therapy is not accompanied by music. Popular aerobics is quite similar to dance therapy, whereas it mainly targets at the general public rather than clinical patients or professional athletes. Due to the strong sense of rhythm and graceful dancing movement, popular aerobics is preferred by the general public. However, most people in China engage in popular aerobics just in order to lose weight, constantly inducing people to make connection of popular aerobics with obesity. Nevertheless, the function of popular aerobics is not simply keeping people fit. In the pioneering book 
"Evolution and Ethics", Yan Fu² proposes reasons why Westerners dominate the world in modern times mainly lie in Westerners' superior wisdom and their physical strength. Hence, he strongly advocates the significant benefits of physical education to Chinese nationals. Why Westerners are so passionate about sports and dancing? This paper investigates the profound philosophical connotations of popular aerobics and thus analyzes justification for Westerners' passion about sports and dancing.

Generally speaking, current research on sports mainly focuses on a specific level rather than provide a holistic view of sports per se. For example, exercise physiology emphasizes the impact of physical activity on cardiovascular system, respiratory system, digestive system, joint bones, and fatigue, whereas exercise psychology focuses on mental health, especially the effects of aerobics on positive activated effect (Reed et al., 2006, 2009), anxiety (Wipfli et al., 2008), body image (Hausenblasa et al., 2006), cognitive function (Etnier et al., 1997), executive function (Chen et al., 2011), depression (Craft et al., 1998 \& Wang et al., 2008) and self-concept (Huang and Guo, 2008). However, research on the overall impact of physical exercises is scanty, so is research on the benefits of physical exercises from the philosophical perspective. Li (1998) studies the philosophical value of sports, but it focuses on competitive sports. In contrast, this paper focuses on leisure sports, namely popular aerobics, which are widely practiced by the general public, and examines the philosophical connotations in depth. It aims to break the prejudice that Chinese people fundamentally hold against popular aerobics, with a purpose of advocating a correct understanding of popular aerobics.

The main reasons why this paper focuses on popular aerobics with the case of Lesmills lie in the following five aspects. First and foremost, Lesmills has a complete system of fitness curriculum, consisting of exercises of different intensities and difficulties. Hence, exercisers can choose the curriculum according to their own preferences and physical strength. For example, spinning is suitable for exercisers interested in cycling. It requires no foundation in dancing. Bodyvive is easy to learn. It requires less strength, which is suitable for novices. Bodybalance with soothing rhythm is mainly preferred by elderly exercisers. Bodypump is easy to learn, but muscle load is required. This exercise helps to shape the body quickly. Bodycombat and bodystep are fast-paced and are of high intensity, which require high cardiopulmonary functions and are more suitable for young exercisers. Bodyjam is graceful and is of moderate intensity. It is characterized by a certain degree of difficulty, which is suitable for young exercisers with

\footnotetext{
${ }^{2}$ Yan $\mathrm{Fu}$ is a Chinese scholar and translator, most famous for introducing western ideas, including Darwin's “natural selection”, to China in the late 19th century.
} 
certain foundation in dancing. Secondly, all fitness instructors of Lesmills must be in possession of professional qualifications to deliver training courses. Meanwhile, regular professional training and assessment are conducted to ensure the quality of Lesmills courses. Previous research has revealed that qualified community fitness instructors can significantly promote people's initiative in participating in physical exercises. Thirdly, compared with traditional Chinese popular aerobics which focus on technical and rhythmic aspects, Lesmills attaches more importance to muscle control and strength, especially the control of core muscle strength. Self-control is not only an important manifestation of an individual's executive function, but also the premise of an individual's adaptability to society. Fourthly, traditional popular aerobics usually lack systematic organization and regularity, often leading exercise classes to vary largely among different fitness instructors, whereas Lesmills has a kind of systematic fitness classes, the body movements of which are designed regularly by professional teams taking into account of the latest pop music and dance movements. Lesmills professionals put specific emphasis on the coordination of music, technology and power. Furthermore, in order to facilitate learning, Lesmills choreographs progress from easy level to difficult level. It eliminates transitions from simple movements to combined body movements, in order to ensure that exercisers are able to grasp the essentials of body movements while maintaining the consistency of dance movements. Last but not least, traditional popular aerobics generally have fixed routines, so that exercisers can easily get bored whereas Lesmills constantly updates its courses, at an interval of a quarter of a year. On one hand, Lesmills can stimulate different muscles, avoiding the problem of muscle resistance caused by practicing the same movements over a long time. On the other hand, Lesmills exercises can help exercisers avoid getting weary. Therefore, it contributes to exercisers' long-term participation in physical exercises, and finally developing a habit of exercising. Previous research has indicated that only through long-term persistence can exercisers obtain their expected results. Lesmills has gained popularity all over the world shortly after its appearance in New Zealand. It has become extremely popular in gyms across China, as it reflects the culture of the Western world instead of being a simple fitness exercise. Therefore, this paper intends to examine the philosophical connotations of popular aerobics using Lesmills as an example.

\section{Philosophical Connotations of Popular Aerobics}

From the view of time and space, popular aerobics abolish time and space, putting emphasis on immersing in current existence and the active participation of body and soul, to experience the self- integrity and sense of existence. Exercise makes the body full of strength, strengthen self-will, 
enabling individuals to have the spirit and the courage to challenge themselves. Popular Aerobics also enhance sensibility to avoid individual sinking into the rigid rule of soul and rationality.

\section{Self-Integration and Sense of Existence}

The life of an individual can be divided into two parts, namely work and leisure. According to Thomas Moore, when people integrate work with the unique aspect of their soul, they can realize that the results gained from nature and intelligence are satisfactory, which can cure all wounds. Whether the work of an individual deserves respect of others depends on the spirit rather than the behavior that he conducted to complete the work. Meanwhile, the quality of leisure depends on the pleasure gained by the soul. Otherwise, it is just a waste of time regardless how high a consumption level an individual is on. Having a good rest is for the sake of doing a better work. An individual with high-quality leisure time is likely to perform better in his career, while popular aerobics happens to be an activity that enhances the quality of leisure time.

The modern society liberates people, with people being able to command their own fate, success or failure. People can become economically independent as long as they work hard. Despite providing people with more freedom, the modern society pushes individuals to become slaves to work, a tool to achieve economic goals. Although this fact to a large extent contributes to the rapid development of social productivity, it also turns people into slaves for the machines manufactured by people themselves. It is easy for individuals to feel their insignificance and weakness once they work with a purpose beyond their thoughts, while modern technology, mass media, network, and digitization further exaggerate the meaningless and rootlessness of people. According to the renowned British sociologist Bauman, mankind is "living in fragments". Therefore, no individual moment is linked together with other moments, which means there is no center of himself nor any identity, failing to make oneself integrated.

Although the modern society fully enjoys freedom of speech, people lack the ability of thinking independently, i.e., being completely free from external interference and in possession of the ability to express pure personal ideas. Kierkegaard argues that "people are irrational, for they never make full use of their freedom of thinking, but continue to pursue what they do not have, which is the freedom of speech". In order to get rid of loneliness and sense of loss, people succumb to those emerging authorities, forcing themselves to adapt to socially accepted behavior patterns, while losing their own identities. 
Fromm argues that people's failure in doing activities spontaneously and expressing their true feelings and thoughts will result in failure in expressing themselves sincerely in front of others, which directly leads to a sense of inferiority and cowardice.

Sport is a culture of identifying self-existence and personal strength. Lesmills is popular for its establishment of a curriculum, which enables exercisers to gain the flow as Csikszentmihalyi proposes. People who have participated in physical exercises are aware of the fact that if the movement is too difficult with respect to their ability, they can easily get bored, whereas if the body movement is too complicated, they will feel anxious and frustrated. Lesmills, though with a certain degree of difficulty, contains a transition from simple to complicated combinations of actions, and repetition is considered to ensure that individuals are able to master complicated actions through constant practice. Moreover, Lesmills dance class curriculum places great emphasis on coordination and cooperation between music and movement, which is based on the latest popular music. When an individual fully immersed in passionate music, he can experience the perfect combination of "body" and "soul" through limb movements and the coordination with music, doing all they want to enjoy the individual moment and experience the sudden "bloom" of his life. This is the optimal state, in which individuals can experience a rapt feeling of being fully immersed in an activity, losing to themselves, others and time perception to experience the notion "this moment is eternal". It reconstructs and integrates the shattered sense of space that people hold. In this state, people can get access to the state of eternity. An individual is no longer concerned about the views of others, only enjoying the music and releasing themselves. People once are engaged in performances or competitions know that if they try to win the applause of audiences, the effects are often unsatisfactory, whereas when they no longer focus on how audiences view them, they tend to be distinguished. For example, people often hear athletes saying after being awarded, "I just enjoy the sport". Studies have shown that the happiest time people perceive is not when they are unconsciously quiet, but when they fully throw themselves into a selfless spirit of challenging.

Zhang (2013) summarizes the abovementioned arguments as the "flow theory", a special self-enjoyment state that occurs naturally, with a special status of total self-immersion. Jackson and Eklund (2002) indicates that physical activities are most likely to create flow experience. Since Lesmills puts emphasis on the coordination among music, movement, and rhythm, together with certain challenges of techniques and skills as well as the clear goal and feedback given by fitness instructors, it is easier for people to get the flow experience. In the flow experience, people tend to forget the existence of themselves, the ticking away of time, the running of the world, 
as well as their internal feelings. Likewise, people can demonstrate their full ability, which is not deliberately cultivated, but gained from the flow of selfconsciousness, enabling people to automatically achieve the optimal result.

\section{Abundance in Strength and Courage}

The training of will must be based on the physical and mental suffering of the individuals. China's famous biologist Tang Peisong admitted that the reasons why he was able to overcome many difficulties and setbacks in the course of study and work as well as maintaining excellent athletic competitive attitude and spirit were deeply rooted in the compulsory sports system during his eight-year-stay at Tsinghua University. In a discussion of child's education, Montaigne argued that strong muscles are prerequisites of getting a strong heart. Only after working habits are fully developed can one cultivate the habit of enduring sufferings; in order to make him endure the pain of future joint stand off, stomach and torment of the disease, physical exercise is of great necessity. It is reported that the Japanese have established lots of "sunshine" kindergarten, with children under six naked when attending school, aiming to cultivate their tastes and strengthen their will, which is regarded as the continuation of carrying forward the spirit of Bushido in contemporary Japan.

Mao Zedong believes that will is the pioneer in life and career. Mao Zedong attached great importance to physical exercise for will training. He himself adhered to lifelong exercise, for instance, cold bath, sunbath, air baths, swimming, hiking, sleeping in open areas, long walks, boxing and gymnastics, almost all of which are related with the honing of will. Thus, when meeting with educators in Hunan Province in 1951, Mao Zedong said, "those who fail to exercise are not in the position to talk about revolution". It is known to all that everyone has some underlying fear in his mind. Some people worry about bankruptcy. Some people are scared of cancer, insomnia, and other diseases. Some others are in fear of rejection. Kierkegaard once wrote that "adventure, in the highest sense, is to be precisely aware of the person himself". An individual, therefore, must have the courage to experience spiritual sufferings, through self-reflection, and then go through Nirvana rebirth, after which he can truly become spiritually mature and creative.

Even in creative individual itself, there exists fear of moving forward. To some extent, the majority of people want to perform better. To satisfy the desire, enjoy the fun of struggle and do our best to seek change, individuals must have the courage to confront difficulties and setbacks. There is no making without breaking, creating means breaking old traditions, getting rid of the past orderly relations which people have get accustomed to, and finally create new relationships. Every truly creative activity means getting a 
higher level of self-awareness and personal freedom, which requires great courage, for people tend to retreat easily in front of the customs. Customs means stability, but sticking to convention may lack innovation and vitality. Both individuals and society are sure to be innovative and vital, once development and progress are to be achieved.

It needs courage to bravely face the fear in the mind. In modern society which is in absence of physical labor, people should especially gain strength through participation in physical exercise, to cultivate the courage and face future challenges. The more powerful an individual is, the more positive attitude he tends to hold and is more likely to accept himself. Rousseau indicates that the more robust a body a person owns, the more likely is he to become intelligent and knowledgeable. Goethe also pointed out the importance of body strength for creativity, saying, "In the past there have been a period of time when people in Germany regarded the genius as little skinny hunchbacks, but I would rather see genius being robust and full of strength".

Plato once wrote in "Republic" that whether horses, dogs, or other animals will not be brave regardless of being full of vigor. Have you ever noticed that high-spirited and emotional state is irresistible and never to be conquered? Will you be fearless and invincible once with it?

The experiences of sports winners have shown that only when the mind, body and action combined together can goals be achieved. In other words, in order to get a good performance, people must have the right attitude, a certain degree of sport skills, together with robust body and the courage facing challenges.

China's traditional culture regards gentle and frail as beauty, leading popular aerobics to focus too much on rhythm and techniques, while ignoring the training of muscle strength. In contrast, Western culture focuses on the beauty of muscle and strength, with Lesmills putting emphasis on the training of muscle strength and lines, especially the strength of abdominal muscles, back muscles, arms and legs. Core strength of the body is of significant use to various activities. Experiences have shown that improving technology alone is not enough to improve athletic performance, whereas core strength training is needed. In recent years, the United States and other developed countries have put particular emphasis on core strength training, which has effectively improved the physical fitness and performance of athletes. An individual will be equipped with the strength and courage to face challenges only with a body full of power. Lesmills introduces barbell into group fitness classes, combined with changes in music, rhythm and movement, shifting the original emphasis on rhythm to group fitness classes coordinating with strength, technique and rhythm. In Lesmills dance classes, especially bodypump exercises, body combat, CX-WORX (core strength 
training), every workshop is a challenge, leading individuals to constantly challenge their mental and physical extremes and limits. Obviously, it is easy to give up, but only insisting until the end can people achieve the selfefficacy. However, insistence requires great courage, which needs the support of the will. Lu Xun ${ }^{3}$ once said that those who keep running to the finish line even if they knew they would be the last are the real backbone of China. Exercises combine will and rationality together. When they are trained, they tend to control the desire. When thinking with rational, the will is fighting for the rationality and obeying the command in order to complete the instructions bravely. An individual tends to gain more confidence each time he completes a challenge. During the course of challenge, members and fitness instructors, and members themselves constantly motivate each other. Furthermore, the joy and heartiness of the glimpse between the winners after the completion of the challenges can only be experienced by people personally involved in the process.

\section{Increment in Sensibility}

Although there are conflicts in the philosophy about whether the knowledge originated in the rational or sensory perception, there is no doubt that the sensory perception of happiness is crucial for subject well-being, making individuals romantic, poetic and fun. Moreover, it is the recognition of the sensory world by Aristotle that greatly encouraged the development of the natural sciences. Throughout the course of human history, it is the emphasis on emotional world that contributes to the advancement of rationality. It is acknowledged that too much emphasis on emotion will make life dangerous whereas too much emphasis on rationality will inevitably make life boring. Therefore, only with coordinated development of rational and emotional will a strong nation be built.

Rousseau believes that since everything going into people's minds through human sense, the initial understanding of people is an emotional understanding, which is the basis for the understanding of rationality. Hence, he believes feet, hands and eyes are the initial philosophy teachers of human beings. In order to learn, individual needs to exercise his limbs, feeling and various organs, because they are tools of human intelligence, thus in order to make full use of these tools, people who uses these tools should be robust and health. Therefore, the real understanding of human does not independently exist. By contrast, the good physique is a basis for good and

\footnotetext{
${ }^{3} \mathrm{Lu}$ Xun is a leading figure of modern Chinese literature. Writing in Vernacular Chinese as well as Classical Chinese, Lu Xun was a novelist, editor, translator, literary critic, essayist, and poet.
} 
thinking abilities. So referring to the famous quote of Roman poet Juvenal, "a sound soul dwells in a sound body".

If people say that science represents the human rationality, then the sport represents the human sensibility. Undeniably, the development of modern science and technology plays an unparalleled role for advancement of human society, but it also resulted in the separation of the human body and soul. Human intelligence and social organization lead the regression of people's physical and spiritual status. The development of rationality brings advanced technological civilization, but with the shrinking of human living space, people constantly staying at home to enjoy the cage-liked life in the high-rise buildings, the severe problem of muscle hunger and dysfunction tends to rise. In modern society, cars, trains, airplanes, cell phones and other electronic devices flooded every corner of the world, which make the human emotional life choked. Comfortable modern life makes the valuable part of individual organs become weak and even deteriorated. In the process of physical fitness, individual needs to use his hand, feet, ears, eyes, and a variety of organs constantly, which not only improves the coordination and balance of the body, but also improve the ability of observation and imitation and make the sense more acute.

Olympia Games commenced in Ancient Greece as early as 776 BC. The highly advancement of Ancient Greek mathematics and philosophy were primarily attributed to the robust body. Observing at the ancient Greek sculpture, it can be concluded that they put particular emphasis on the beauty of muscle and strength. Danner points out that in the eyes of the ancient Greeks, the ideal figure is not simply the mind which is good at thinking or feeling, but the naked body with descent blood relationship, being well developed, well-proportioned and good at a variety of sports. Philosopher Socrates, Plato, Aristotle, the poet Timothy Craig Dion and playwright Sophocles, Euripides Adams, were all very famous athletes at that time. Before mathematician Pythagoras discovered the Pythagorean theorem, he used to be a good boxer, who won the Olympic boxing champion. Besides, even when later in the school, he also taught boxing and fencing in addition to mathematics courses.

Lesmills is a comprehensive fitness class with strong artistic features, which emphasizes the seamless combination among music, body movements and muscle strength, trying to make each action reflect the beauty of the human body and the lines of muscle strength. It is the practical effort of the liberation of the human emotion under pressure of rationality that involves rich aesthetic content. In the process of keeping fit, people are committed to restore and maintain the natural properties of being a living creature and thus indulging themselves in a state of life aesthetic. The true meaning of life together with the aesthetic pleasure is realized in such physical strength 
expression and the hone of will. Socrates believes that knowledge is the supreme good, whilst Nietzsche points out that people cannot rely on the truth of life for living. Individual life is constantly produced and destructed. If people explore the meaning of life with true or moral vision, people tend to get depressed and lose confidence in life, which is the tragedy of human life. Those who have a deep understanding of life can only seek a shelter from art in order to avoid the quagmire towards hedonism and nihilism. Art leads to the free development of human life instinctively and the emotion repressed by rationality, which is the highest form of instinct sublimation of human life. It is beauty that makes life worthy, even the greatest scientist Dirac has strongly recommended that making an equation with beauty is even more important than making it with experiments. The love of beauty originates from the love of life, for beauty is an unrestrained state of passion. However, only those who are healthy gain the beautiful life. In Lesmills, graceful movements greatly enrich and deepen the experience of beauty, enabling people to feel the perfect combination of music and limbs, and eventually turning the pain of life into happiness through the fondness and indulgence of music and sport. People tend to seek life instinct sublimation in the process of exercising while looking beyond the soul in the pursuit of music. Individual can feel the freedom and release of his body, mind and spirit, enjoying the pleasure of exercising and sports, as well as returning to the instinct of life, and eventually reach the realm of harmonization of body and soul. Moreover, a person with a noble soul is unlikely to appreciate his own life only. Instead, he can certainly realize the truth that all creatures are from an organic whole and they are homologous, and thus reaching the sublimation of soul.

\section{Conclusion}

This paper examines Lesmills from a specific perspective, which is primarily based on the philosophical connotation of public fitness. A great deal of research pertains to the effects of physical exercises on promoting physical health and mental well-being. Nevertheless, most research is confined to exercises for special needs, e.g., medical treatment or athletic performance improvement, somehow leading people to form misunderstandings on physical exercises. It is noteworthy that most people subconsciously regard popular aerobics as a form of "playing” or "dancing”, i.e., a form of exercise no more than entertaining. Digging into the history of Chinese civilization, people's limited understanding of popular aerobics may result from the fundamental ideas that exist in Chinese agriculture civilization for thousands of years, namely an ideology that values agricultural activities while neglects trade, a mentality that values literary talents while neglects martial arts. Such values eventually cultivate the 
nation to regard "being weak" as beauty. Similarly, China is a country with a relatively introverted personality. It favors fair and reasonable results and does not avidly engage in adventures. In contrast, Western civilization is a marine civilization, featured with fondness for adventure and conquest. Besides, commercialism further contributes to Western civilization's preference for competition and thus induces Western culture to put significant emphasis on physical exercises, as remarkable courage is needed in sailing, adventure and conquest. For Westerners, every adventure and conquest is a process of battling with death alone at first, getting familiar with it afterwards, and winning the battle eventually.

Suppose that an individual has not done much physical exercises, then it is less likely that his body or soul is fully developed. Similarly, by what means will he be able to overcome his lack of strength and low spiritual self-esteem? How can he embody "free nature" in the cooperative social networks? This paper discusses the connotation of Lesmills from a philosophical perspective. It mainly argues that people should, on a cognitive level, change their prejudice against popular aerobics. People should establish an impersonal and scientific understanding of popular aerobics. If people who are interested in popular aerobics can actively and persistently practice, then they stand a high chance in promoting a harmonious development among people, society and nature, making scientific exercises a way and a realm of life. More precisely, only people who are both physically healthy and mentally healthy can contribute to the society. In conclusion, popular aerobics, especially Lesmills have profound value and should be urged. However, due to individual differences, there is no exercise that is suitable for all. Thus, people should make choices based on individual interests and hobbies.

\section{References:}

Stark, A., Newton, M. (2014), A Dancer's Well-Being: The Influence of the Social Psychological Climate During Adolescence. Psychology of Sport and Exercise, 15, 356-363.

Bandura, A., Schunk, D.H. (1981), Cultivating competence, self-efficacy, and intrinsic interest through proximal self-motivation. Journal of Personality and Social Psychology, 41, 586-598.

Bing, Z., Yang, Z. (2012), Expolre The Origin of the Rise and Fall of Ancient Greek and Medieval Sports: Based on the Physical and Recreational Aspects [J] Sports Science, 32 (1), 88-95.

Bradley M. Wipfli, C. D. R., D. M. Landers (2008), The Anxiolytic Effects of Exercise: A Meta-Analysis of Randomized Trials and Dose--Response Analysis. Journal Of Sport \& Exercise Psychology, 30, 392-410. 
Craft, L. L., Landers, D. M. (1998), The Effect of Exercise on Clinical Depression and Depression Resulting from Mental Illness: A Meta Analysis. Journal Of Sport \& Exercise Psychology, 20, 339-357.

Csikszentmihalyi. Cardiac Life Flow [M]. CITIC Publishing House, 2009.

Diener. E., Suh. E, Lucas. R. et al. (1999), Subjective Well-Being: Three Decades of Progress. Psychological Bulletin, 125(2), 276-302.

Etnier AL, Salazar W., Landers, DM, Petruzzello SJ, Han M, Nowell P. (1997), The Influence of Physical Fitness and Exercise upon Cognitive Functioning: A Meta-Analysis. Journal Of Sport \& Exercise Psychology, 19, 249-277.

Fromm. Escape Freedom [M]. International Cultural Publishing Company, 2002.

Goldwater, B. C. Collins. M. L. (1985), Psycho Logic Effects of Cardiovascular Conditioning: A Controlled Experiment. Psychosomatic Medicine, 47(2), 174-181.

Hausenblasa E., Fallon, H. A. (2006), Exercise and Body Image: A MetaAnalysis Psychology \& Health, 21(1), 33-47.

Hayes, D., Ross, C.E. (1986), Body and Mind: The Effects of Exercise, Overweight, and Physical Health on Psychological Well-being, Journal of Health and Social Behavior, 27, 387-400.

Hegel, Little Logic [M] Beijing: Commercial Press, 2012.

Justy R,Deniz, S. O., (2006), The Effect of Acute Aerobic Exercise on Positive-Activated Affect: A Meta-Analysis. Psychology of Sport and Exercise. No.7. 477-514.

Justy R., Sarah, B., (2009), The effect of Regular Aerobic Exercise on Positive-Activated Affect: A Meta-Analysis. Psychology of Sport and Exercise. No.10. 581-594.

Kierkegaard S., Trembling With Anxiety [M]. Tianjin People's Publishing House, 2007.

Li, L., Barbaric Civilization: Philosophical Declaration of Sports [M] Chinese Society Press, 1998.

Lox. L., C. McAuley. E. (1995), Exercise as An Intervention for Enhancing Subjective Well-Being in An HIV-1 Population. Journal of Sport \& Exercise Psychology, 17, 345-362.

Nietzsche's The Birth of Tragedy [M]. Yilin Press, 2011.

Plato. Utopia [M]. Commercial Press, 2012.

Rang P. S., Existentialism Is A Humanism [M]. Shanghai Translation Publishing House, 2012.

Robert A., Mechikoff S. G. E., A History and Philosophy of Sport and Physical Education: From Ancient Civilizations to the Modern World [M]. New York: McGraw-Hill, 2006. 
Rousseau's Emile (upper and lower volumes) [M] Beijing: Commercial Press, 2012.

Russell's History of Western Philosophy (upper and lower volumes) [M] Beijing: Commercial Press, 1963.

Snyder E.E, Spreitzer E. (1974), An Involvement in Psychological WellBeing. International Journal of Sports Psychology, 5, 28-39.

Spence JC,B.J.C.N. (2006), Self-Efficacy and Physical Activity: A Quantitative Review. Journal of Sport \& Exercise Psychology, (28), 172. 